\title{
Gas Adsorption on the Surface of Ferromagnetic Pd Nanoparticles*
}

\author{
Yojiro Oba ${ }^{\dagger}$ and Tetsuya Sato \\ Department of Applied Physics and Physico-Informatics, Graduate School of Science, \\ Keio University, Yokohama, Kanagawa 223-8522, Japan \\ Takenao Shinohara \\ Image Information Research Unit, RIKEN, Wako, Saitama 351-0198, Japan \\ (Received 14 October 2005; Accepted 9 April 2006; Published 25 April 2006)
}

\begin{abstract}
The appearance of ferromagnetism in the surface regime of Pd nanoparticles was recently reported [Phys. Rev. Lett. 91, 197201 (2003)]. To experimentally confirm the surface magnetism, the effects of $\mathrm{O}_{2}$ gas adsorption on the $\mathrm{Pd}$ nanoparticles are studied based on the measurements of the coverage and magnetization. The surface ferromagnetism was weakened in the samples fully covered with $\mathrm{O}_{2}$ gas. However, the ferromagnetic moment remained after the exposure to $\mathrm{O}_{2}$ gas. This result shows the ferromagnetism existing in the internal regime in addition to the surface magnetism. [DOI: 10.1380/ejssnt.2006.439]
\end{abstract}

Keywords: Magnetic measurements; Magnetic phenomena; Palladium; Powders; Magnetic properties of nanostructures; Nanoparticles; Ferromagnetism; Oxygen

\section{INTRODUCTION}

The surface of materials shows interesting nature because of the reduction of coordination number or the change in symmetry. Novel physical properties may arise from the two-dimensional (2D) characteristics appearing on the surface. Thin film has been regarded as the optimal shape for the study of the 2D physical properties. However, substrates, which are necessary to prepare thin films, cause some problems, such as the orbital hybridization, the mismatch of the lattice constant, and the atomic diffusion between films and substrates. Therefore, the intrinsic 2D physical properties may be insufficiently studied using thin films. On the other hand, nanoparticle is suitable to study the intrinsic $2 \mathrm{D}$ physical properties, because of the large surface areas ratio to total volumes without substrates.

Palladium is a good system to study the $2 \mathrm{D}$ magnetism on the surface. Several theoretical studies have predicted the appearance of ferromagnetism on $\mathrm{Pd}$ thin film [1-3]. However, experimental studies have reported only negative results $[4,5]$. The first evidence for the ferromagnetism in $\mathrm{Pd}$ was recently discovered in the clean and free-standing nanoparticles $[6,7]$. These studies showed that the only (100) facets of the nanoparticles are ferromagnetic and the other region is paramagnetic like bulk $\mathrm{Pd}$. This indicates the appearance of 2D magnetism in the Pd nanoparticles. However, the mechanism for the appearance of ferromagnetism in $\mathrm{Pd}$ nanoparticles is insufficiently understood, since the ferromagnetic region has not been directly observed. In this study, we discuss the ferromagnetic region in $\mathrm{Pd}$ nanoparticles based on gas adsorption experiment to clarify the origin of ferromagnetism. This is because the adsorbed gas can change electronic structure only on the surface, and thus the surface

* This paper was presented at International Symposium on Surface Science and Nanotechnology (ISSS-4), Saitama, Japan, 14-17 November, 2005.

†Corresponding author: oba@az.appi.keio.ac.jp magnetization can be separately evaluated.

\section{EXPERIMENTAL}

Pd nanoparticles were prepared by a gas-evaporation method in Ar gas (99.9999\% pure), which was conditioned through a gas filter. The experimental detail is described elsewhere [7]. The initial vacuum was $2 \times 10^{-9}$ Torr. The nanoparticles were collected and sealed in a quartz tube attached to the bottom of the chamber. Since the samples were never exposed to air, the nanoparticles could have the clean and free-standing surface. Gas adsorption was performed after the evaporation. Oxygen, which is strongly adsorbed on the surface of $\mathrm{Pd}$, was chosen as an adsorbed gas, because we expect to drastically change the surface electronic state with the $\mathrm{O}_{2}$ gas adsorption. The coverage was controlled by the change in gas partial pressure and monitored by a crystal oscillator. The size and morphology of samples were observed by transmission electron microscope (TEM). The crystal structure was characterized with X-ray diffraction (XRD). A superconducting quantum interference device (SQUID) magnetometer was used for magnetic measurement at temperatures between $5 \mathrm{~K}$ and $300 \mathrm{~K}$.

\section{RESULTS AND DISCUSSION}

The polyhedron Pd nanoparticles with flat surfaces, such as octahedron, decahedron, and truncated ones were obtained (Fig. 1). The octahedron and decahedron correspond to fcc single crystal and multiply twinned (MT) structure, respectively. Their surfaces consist of (100) and (111) facets. A typical size distribution of Pd nanoparticles is shown in Fig. 2. The average diameter of $\sim 20 \mathrm{~nm}$ was determined by fitting a log-normal function into the size distribution for each sample.

The time dependent mass of $\mathrm{Pd}$ nanoparticles in the sample preparation process is shown in Fig. 3. After the evaporation of $\mathrm{Pd}$, the remarkable increase in the mass, 

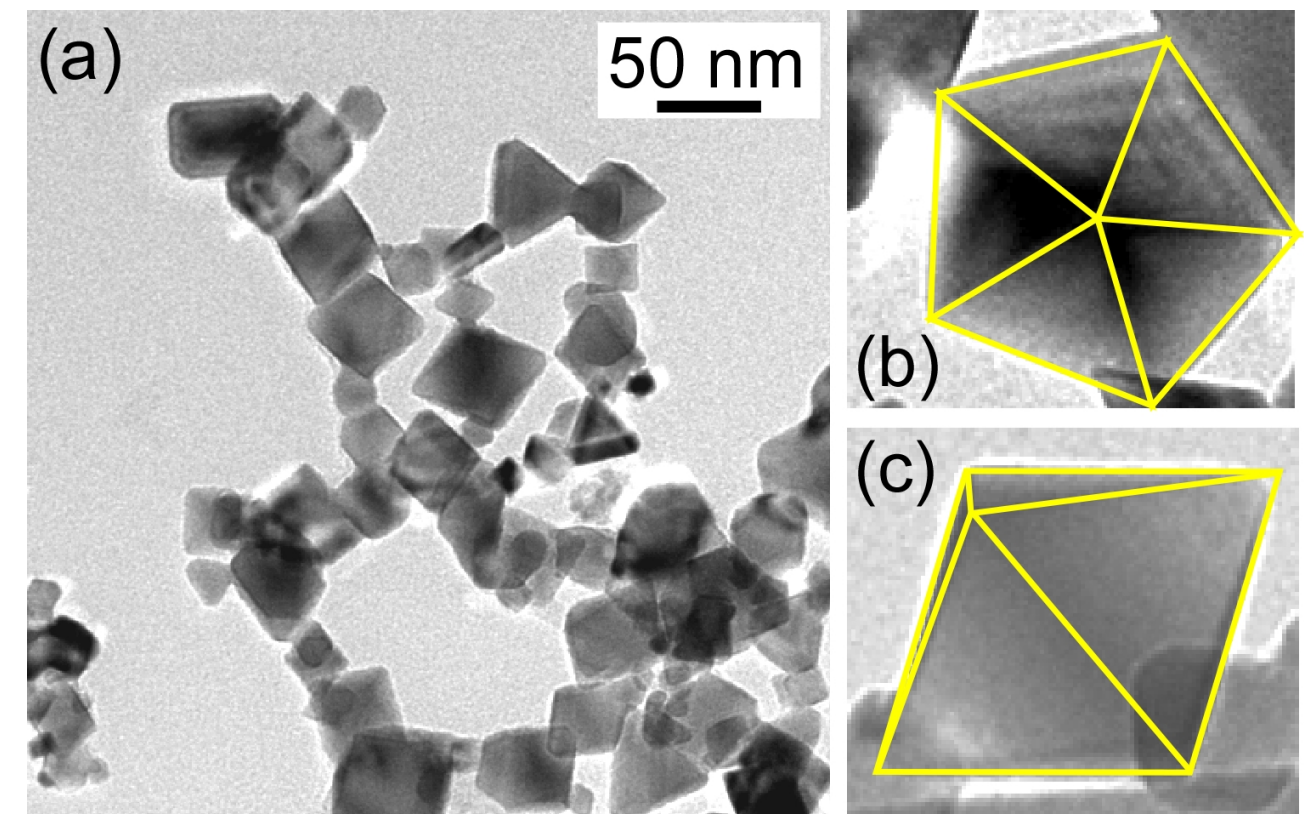

FIG. 1: (a) TEM image of Pd nanoparticles. (b) Enlarged TEM image of an octahedral particle. (c) Enlarged TEM image of a decahedral particle.

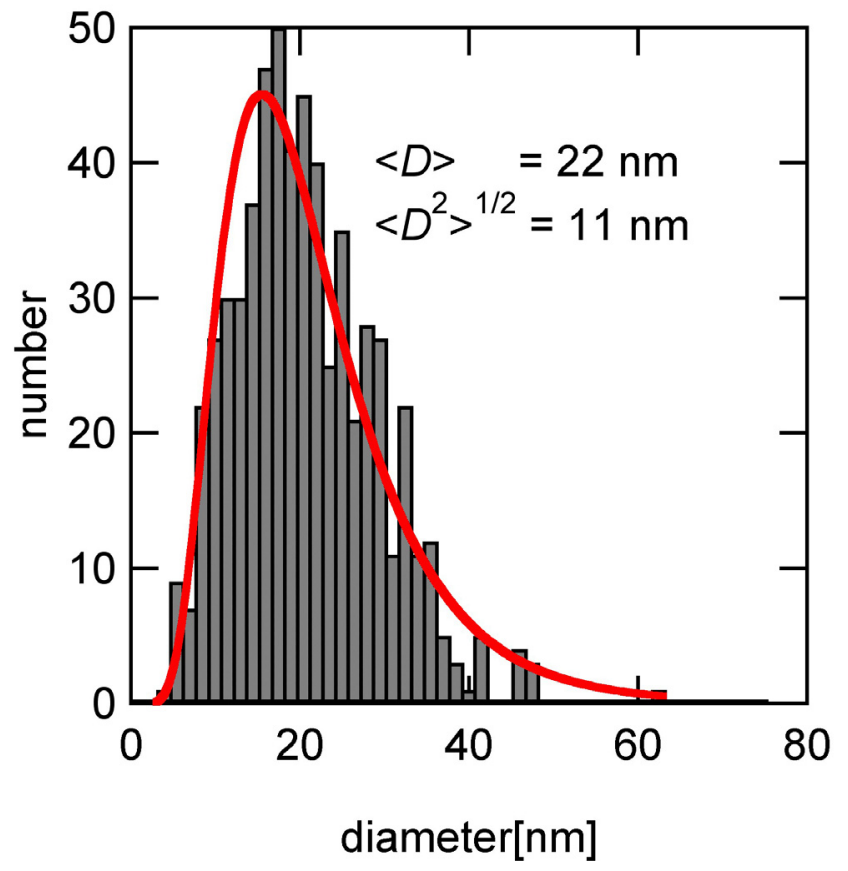

FIG. 2: Size distribution of a typical sample. $\langle D\rangle$ and $<D^{2}>^{1 / 2}$ are the average diameter and standard deviation, respectively.

which is caused by the deposition of nanoparticles, was observed. As we injected $\mathrm{O}_{2}$ gas into the chamber (arrows in Fig. 3 indicate the injection of $\mathrm{O}_{2}$ gas), the mass of nanoparticles once decreased and then increased and finally approached to a constant. This behavior was not observed when Ar gas was injected instead of $\mathrm{O}_{2}$. Thus, we can estimate that the first step decrease is caused by the desorption of Ar gas, which is trapped among the nanoparticles, and $\mathrm{O}_{2}$ gas brings about the desorption.

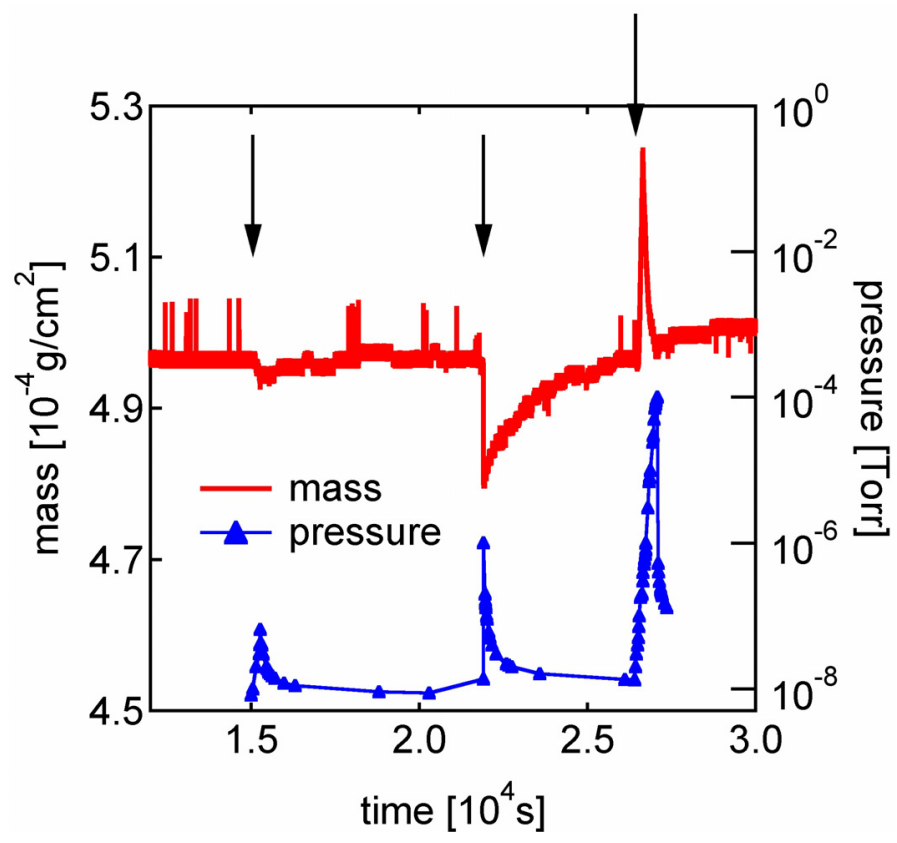

FIG. 3: Time dependent mass of samples. Arrows indicate the injection of $\mathrm{O}_{2}$ gas.

The following increase corresponds to the adsorption of $\mathrm{O}_{2}$ gas. The coverage was estimated to be $\sim 4 \mathrm{ML}$ for the $\mathrm{Pd}$ particles held in $\mathrm{O}_{2}$ partial pressure of $1 \times 10^{-6}$ Torr, i.e., nanoparticles are fully covered with $\mathrm{O}_{2}$ molecules.

The magnetization consists of a saturated part and a linearly increasing part as the increase in magnetic field in all temperatures. This shows the coexistence of ferromagnetism and paramagnetism in the $\mathrm{Pd}$ nanoparticles. Therefore, the magnetization can be represented as a simple sum of the ferromagnetic component and the 


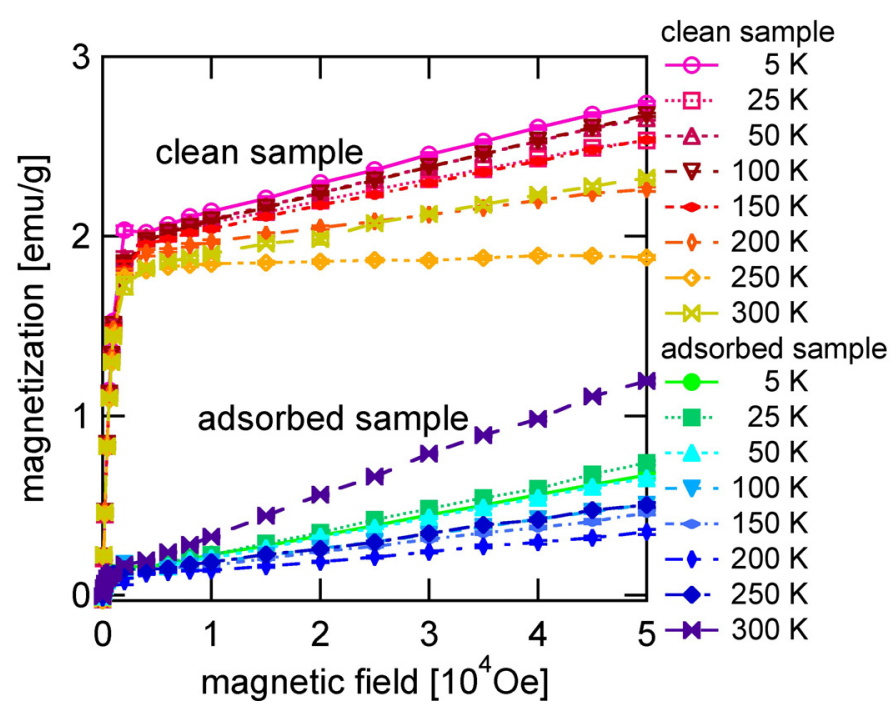

FIG. 4: Magnetic field dependence of magnetization on the samples with clean surfaces and with $\mathrm{O}_{2}$ gas adsorbed.

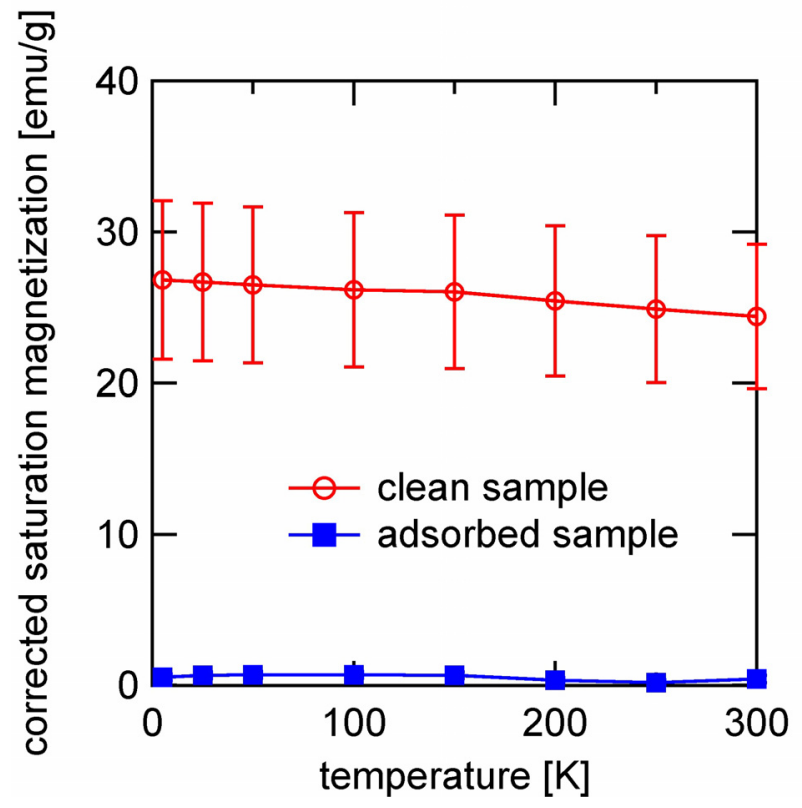

FIG. 5: Temperature dependence of saturation magnetization on the samples with clean surfaces and with $\mathrm{O}_{2}$ gas adsorbed.

paramagnetic one. In order to discuss the effect of $\mathrm{O}_{2}$ gas adsorption on the magnetism, we measured the magnetization of the samples of $\mathrm{Pd}$ nanoparticle with diameter of $\sim 20 \mathrm{~nm}$ which have the different coverage of $\mathrm{O}_{2}$ molecules. The result of magnetic measurement is shown in Fig. 4. As a result, $\mathrm{O}_{2}$ gas drastically reduces the saturation magnetization, i.e., the $95 \%$ decrease in ferromagnetic contribution was observed. On the other hand, the paramagnetic contribution is scarcely affected. However, since the magnetization of the background is similar order to the one of the nanoparticles, the detail of the magnitude of the paramagnetic moment is unclear.

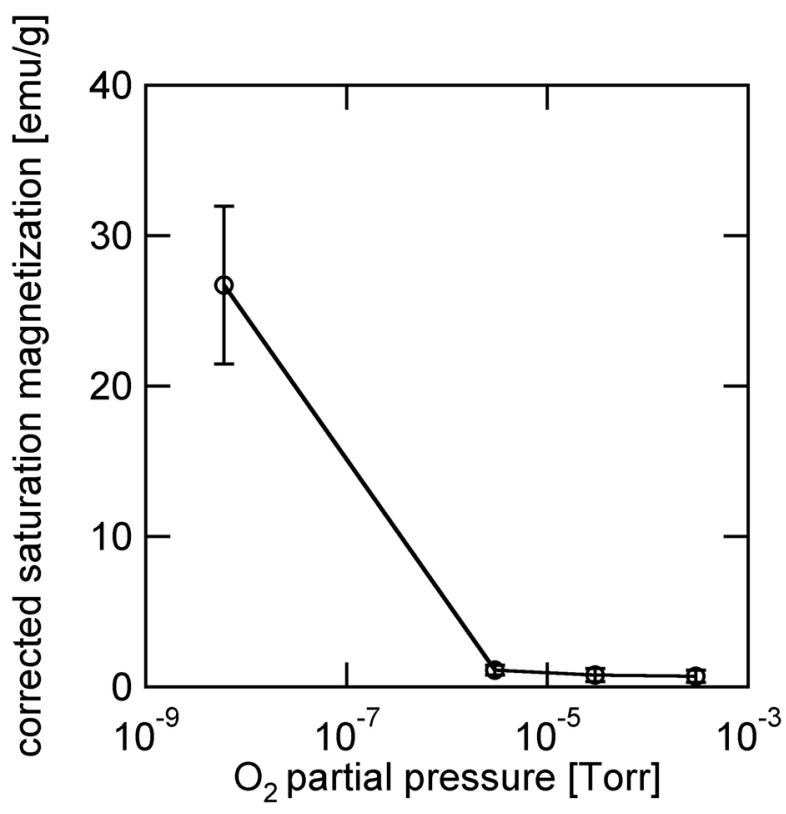

FIG. 6: Partial pressure dependence of corrected saturation magnetization. The plot at least pressure indicates noadsorbed sample.

So far, the ferromagnetism of Pd nanoparticles has been discussed relevant to the shapes or the orientation of the crystal face in the particles. Thus, such a characteristic of each sample should be considered for exact comparison. The previous work indicated that the ferromagnetism appears on the only (100) facets in $\mathrm{Pd}$ nanoparticle [7]. Therefore, in order to correct the difference of the area of (100) facets among the samples, we performed the correction of the saturation magnetization as mentioned below. Each nanoparticle can be regarded as the composite of several pyramids whose apexes are located at the center of the nanoparticle and the facets of the nanoparticle correspond to the bases of the pyramid. A octahedral and a decahedral nanoparticle consist of eight and ten pyramids with a base of (111), respectively. Truncated ones have the additional six and five pyramids with a base of (100), respectively [8]. The ferromagnetism exists in the only (100)-based pyramids. Therefore, the saturation magnetization can be normalized by the division by the ratio of the volume of the (100)-based pyramids to the total volume. The ratio of the volume of the (100)-based pyramids is evaluated from the average diameter and dimensions of the (100) facets for each shape. We measured these values from TEM images and corrected saturation magnetization by the evaluated ratio of the (100)-based pyramid. The further details of the correction procedure was described in Ref. [7]. The corrected saturation magnetization remains even at room temperature and slightly decreases as the increasing in temperature (Fig. 5). The corrected saturation magnetization also clearly decreases accompanied by the $\mathrm{O}_{2}$ gas adsorption, although the change in magnetization among the adsorbed samples is scarcely dependent on the $\mathrm{O}_{2}$ partial pressure (Fig. 6). This reveals that the coverage of them is intrinsically saturated in $3 \times 10^{-6}$ Torr, which is consistent with the measurement of coverage using the crystal oscillator. The corrected saturation 
magnetization of $\mathrm{O}_{2}$ adsorbed sample is $\sim 93 \%$ smaller than that of pure sample. This clearly means that the reduction of the saturation magnetization is caused by the $\mathrm{O}_{2}$ gas adsorption, and then the ferromagnetism exists in the surface regime of the $\mathrm{Pd}$ nanoparticles. On the other hand, the nonzero magnetization remains even in the fully $\mathrm{O}_{2}$ gas adsorbed samples. This may indicate the ferromagnetic contribution from the core region of $\mathrm{Pd}$ nanoparticles.

Based on the previous paper, the surface ferromagnetism in $\mathrm{Pd}$ nanoparticles has been interpreted in a picture of itinerant magnetism, i.e., the ferromagnetism occurs under the Stoner criterion $N_{l o c}\left(E_{F}\right) I>1$, where $N_{l o c}\left(E_{F}\right)$ is the local density of states at the Fermi energy and I is the exchange integral. On the surface, $N_{l o c}\left(E_{F}\right)$ is enhanced due to the smaller coordination number. In addition, (100) plane has an advantage for ferromagnetism compared with (111) planes in fcc crystal structure because of the smaller coordination number. As a result, the ferromagnetism can appear only on the (100) facets of Pd nanoparticles. According to this scenario, the disappearance of ferromagnetism should be caused by the reduction in $N_{l o c}\left(E_{F}\right)$. The present reduction of magnetization can be explained as follows. First, the 2D properties can be weakened, since the $\mathrm{O}_{2}$ gas adsorption brings about the increase in the coordination number on the surface atoms. Second, the change in the Fermi energy by the electron transfer from $\mathrm{Pd}$ to $\mathrm{O}$ atom can induce the reduction of $N_{l o c}\left(E_{F}\right)$, because of the smaller electronegativity in $\mathrm{Pd}$ than O. Légaré, et al. [9] reported the reduction of $N_{l o c}\left(E_{F}\right)$ in $\mathrm{Pd}$ induced by $\mathrm{O}_{2}$ gas adsorption using UPS and XPS.

The remaining saturation magnetization in the $\mathrm{O}_{2}$ gas adsorbed samples suggests the ferromagnetism exists in the core region of $\mathrm{Pd}$ nanoparticles. Theoretical calculations have proposed some candidate for the origin of the internal ferromagnetism in $\mathrm{Pd}$, i.e., the expansion of the lattice constant(positive strain) [10, 11], twin boundary [12], change in the crystal symmetry [13], and vacancy [14]. The results of XRD show that the $\mathrm{Pd}$ nanoparticle has fcc structure with the lattice constant of 3.89 $\mathrm{nm}$, which is only $0.1 \%$ smaller than that of bulk Pd. Thus, the effects of the expansion of lattice constant and change in the crystal symmetry cannot explain the internal ferromagnetism in $\mathrm{Pd}$ nanoparticles. On the other hand, present samples show the well-defined polyhedrons including MT structures, which contain strain and twin boundary even in the stable state. Therefore, the inhomogeneous strain and/or twin boundary are the possible candidates for the internal ferromagnetism.

\section{CONCLUSIONS}

In conclusion, the surface magnetism of Pd nanoparticles was investigated using the gas adsorption experiment. The ferromagnetism drastically disappears with the adsorption of $\mathrm{O}_{2}$ gas. This is the evidence of the surface ferromagnetism of $\mathrm{Pd}$ nanoparticles. However, the nonzero saturation magnetization remains even after the gas adsorption. This suggests the ferromagnetism existing in the core region of nanoparticles, i.e., the coexistence of ferromagnetism in the surface and internal regimes. The internal ferromagnetism possibly arises from the inhomogeneous strain and/or twin boundary in nanoparticles.

\section{Acknowledgments}

This work was supported by the FY 2002 21st Century COE Program and Grant-in-Aid for Scientific Research from Ministry of Education, Science, Sports and Culture of Japan.
[1] J. Ming, J. Zhu, D. M. Bylander, and Leonard Kleinman, Phys. Rev. B 42, 2874 (1990).

[2] S. Blügel, Phys. Rev. B 51, 2025 (1995).

[3] S. Mirbt, B. Johansson, and H. L. Skriver, Phys. Rev. B 53, R13310 (1996).

[4] R. L. Fink, C. A. Ballentine, J. L. Erskine, and Jose A. Araya-Pochet, Phys. Rev. B 41, 10175 (1990).

[5] C. Liu and S. D. Bader, Phys. Rev. B 44, 12062 (1991).

[6] T. Taniyama, E. Ohta, and T. Sato, Europhys. Lett. 38, 195 (1997).

[7] T. Shinohara, T. Sato, and T. Taniyama, Phys. Rev. Lett. 91, 197201 (2003).

[8] Shozo Ino, J. Phys. Soc. Jpn. 27, 941 (1969).
[9] P. Légaré, L. Hilaire, G. Maire, G. Krill, and A. Amamou, Surf. Sci. 107, 533 (1981).

[10] V. L. Moruzzi and P. M. Marcus, Phys. Rev. B 39, 471 (1989)

[11] H. Chen, N. E. Brener, and J. Callaway, Phys. Rev. B 40, 1443 (1989).

[12] F. Aguilera-Granja, J. M. Montejano-Carrizales, and A. Vega, Solid State Comm. 133, 573 (2005).

[13] E. Hüger and K. Osuch, Europhys. Lett. 63, 90 (2003).

[14] N. Takano, T. Kai, K. Shiiki and F. Terasaki, Solid State Comm. 97, 153 (1996). 